\title{
Circuit
}

Musiques contemporaines

\section{Paysage sonore électronique et canadien : introduction}

\section{Jonathan Goldman}

Volume 19, numéro 3, 2009

Pionniers canadiens de la lutherie électronique

URI : https://id.erudit.org/iderudit/038254ar

DOI : https://doi.org/10.7202/038254ar

Aller au sommaire du numéro

Éditeur(s)

Les Presses de l'Université de Montréal

ISSN

1183-1693 (imprimé)

1488-9692 (numérique)

Découvrir la revue

Citer ce document

Goldman, J. (2009). Paysage sonore électronique et canadien : introduction.

Circuit, 19(3), 5-8. https://doi.org/10.7202/038254ar d'utilisation que vous pouvez consulter en ligne.

https://apropos.erudit.org/fr/usagers/politique-dutilisation/ 


\section{Paysage sonore électronique et canadien : introduction}

Jonathan Goldman

Il y a deux ans, dans l'introduction du numéro de Circuit consacré aux avantgardes musicales en Amérique latine ( «Plein sud», vol. 17, n² 2), j’ai écrit ceci :

Lire l'histoire d'un pays qui n'est pas le nôtre, c'est [...] se livrer à un jeu de correspondances. Nous ramenons les personnages, les lieux et les événements qui y sont décrits à des éléments de notre expérience propre. Lorsqu'il s'agit de personnes, nous cherchons un équivalent parmi nos connaissances directes ou indirectes qui prête certains de ses traits - physiques ou spirituels - à l'objet lu et imaginé.

Si je me permets cette autocitation, c'est qu'elle n'est pas sans rapport avec le thème du numéro actuel. Dans ce même éditorial, j’invitais le lecteur à se livrer à des comparaisons entre les moments significatifs du développement des institutions de la musique contemporaine dans notre pays du Nord et dans des pays de l'hémisphère Sud, histoire de nous permettre d'entrevoir des formes de notre expérience qui, autrement, resteraient dans un angle mort historiographique. Ces formes incluaient la recherche de spécificités culturelles dans chacun des pays examinés, les «jeux des cités rivales » - tant esthétiques que reliés à des conflits d'ordre personnel -, le statut de la musique «officielle» par opposition à ses dérives underground, en passant par les institutions qui rendaient possible l'épanouissement de divers genres musicaux. En ce qui concerne les institutions musicales, l'une d'entre elles s'est détachée des autres par l'envergure de ses activités et la force des personnalités qui y étaient associées, du moins dans la première moitié des années 1960. Le Centro Latinoamericano de Altos Estudios Musicales (CLAEM), au sein de l'Institut Di Tella à Buenos Aires, dirigé par Alberto Ginastera - le compositeur national officiel, s'il y en eut un -, a laissé une empreinte indélébile sur l'imaginaire collectif des adeptes des arts progressifs en Amérique du Sud. 
Où est donc notre CLAEM? On sait que le premier studio de musique électronique au Canada s'ouvrait à Toronto en 1959 (l'University of Toronto Electronic Music Studio [UTEMS]), suivi du McGill Electronic Music Studio (EMS) en 1964, mais ni l'un ni l'autre ne possède la même aura. Si trouver un équivalent exact à un institut comme le CLAEM s'avère certes impossible, confronter ses conditions d'existence avec celles de nos propres institutions constitue une belle entrée dans le vif du sujet, celui de l'histoire de la musique électronique au Canada.

La curiosité éveillée par le numéro latino-américain commandait un numéro qui se pencherait sur l'histoire de l'alliage fructueux dans cette «autre autre» Amérique - la nôtre! - de la musique et de la technologie. Vous l'avez deviné, c'est la mission que se voit confié la livraison que voici, qui n'a pas pour principal centre d'intérêt les grandes œuvres de la musique électroacoustique, mais plutôt les institutions musicales qui ont permis la mise sur pied et l'essor subséquent de la musique à moyens électroniques. Nous nous intéressons aussi, bien sûr, aux gens - généralement des compositeurs, mais aussi des ingénieurs, inventeurs, administrateurs, diffuseurs - qui ont œuvré pour la création de tels locaux au Canada, et qui ont contribué à un héritage méconnu, ponctué de fausses pistes, d'heureuses surprises et de quelques coups de génie.

Toute cette activité foisonnante présuppose l'existence du «studio de musique électronique », cet espace de création qui apparaît au milieu du $\mathrm{XX}^{\mathrm{e}}$ siècle. Ces studios se répartissent en trois grandes catégories: ils sont soit autonomes (donc des «home studios»), soit reliés à des institutions universitaires et à des équipes de recherche, ou enfin, reliés à des studios de production cinématographique. Ce sont trois cas de figures explorés dans ce numéro:

1) Le «home studio»: Otto Joachim (1910), dont les propos sont recueillis dans ce numéro par les soins d'Anne Marie Messier, a monté le premier studio maison au Canada (après en avoir établi deux autres à Singapour et à Shanghai...). Dans ce studio personnel, situé dans le sous-sol de sa maison, il a notamment composé Katimavik, œuvre diffusée en boucle dans le pavillon canadien d'Expo 67 à Montréal; ce cas de figure est également représenté par Gordon Mumma (1935), compositeur et bricoleur à ses heures, qui donne la recette pour faire un studio «indépendant» à des lecteurs de 1964 dans un article reproduit ici en guise de document.

2) Le studio relié à une équipe de recherche universitaire: c'est István Anhalt (1919), qui a fondé les studios des universités McGill et Queens, et dont un entretien avec Matt Rogalsky se trouve dans le présent numéro; de 
même, Nil Parent (1945) a fondé en 1969 le studio de musique électronique de l'Université Laval (SMEUL), premier studio francophone au Canada. Gisèle Ricard, compositrice et archiviste à l'Université Laval, qui a suivi de près la démarche de Parent au sein du SMEUL et du Groupe d'interprétation de musique électroacoustique de Laval (GIMEL), offre une chronologie des événements marquants du studio, du groupe, et de leur fondateur, aussi inventeur de nouvelle lutherie. C'est aussi le dispositif du studio de Barry Truax (1947) de l'Université Simon Fraser à Vancouver, dont une entrevue réalisée par Sylvia L'Écuyer est reproduite ici. Truax a atteint la reconnaissance internationale pour ses contributions à l'informatique musicale et, notamment, pour ses travaux sur la synthèse granulaire en temps réel, utilisée à merveille dans son œuvre Riverrun (1986).

3) Le studio en annexe d'un studio de production cinématographique: c'est un cas de figure défendu par l'article de Réal La Rochelle qui effectue un survol de quelques expériences audiovisuelles réalisées à l'Office national du film du Canada, qui ont contribué au développement du genre connu sous l'appellation de «vidéopéra».

Les studios seraient impensables sans des instruments, compris au sens large d'une nouvelle lutherie qui permet un contrôle fin d'un nombre considérable de paramètres acoustiques et, par conséquent, une sorte d'improvisation par le compositeur en studio. Dans cet ordre d'idées, on ne peut manquer de souligner l'apport de Hugh Le Caine (1914-1977), physicien, inventeur de génie et musicien qui, fort de son expertise en matière de technologie de radar, a contribué de façon décisive à l'effort de guerre de l'armée canadienne durant la Deuxième Guerre, ce qui lui a valu des bourses du Conseil national de recherches du Canada (CNRC). Celles-ci lui ont permis de consacrer de longues années à la conception et la fabrication d'instruments nouveaux. Les fruits de son bricolage - hélas jamais industrialisés ou commercialisés - incluent des instruments curieux tels la «saqueboute électronique » et le «multi-track», des originaux en leur genre. Le Caine - nettement plus inventeur que compositeur - est néanmoins l'auteur de la première œuvre canadienne de musique concrète, la sympathique Dripsody (Rhapsodie pour une goutte d'eau) (1955)'. Nous reproduisons ici des extraits de la biographie de Le Caine écrite par la compositrice Gayle Young, parue simultanément en anglais et en français en 1989, peu diffusée au moment de sa parution, et épuisée depuis un certain temps.

Enfin, pas tout à fait hors propos, le Cahier d'analyse explorera une œuvre mixte; il s'agit de Chute/Parachute (1989) de Michel Gonneville, pour piano et bande, analysée par les soins de Nicolas Gilbert. Il va sans dire que
1. Dont un extrait peut être écouté sur l'excellent site officiel de Hugh Le Caine, préparé par Gayle Young: $<$ http://hughlecaine.com/>. 
ce numéro n'a pas vocation de rivaliser avec l'Encyclopédie de la musique au Canada ni - tâche impossible - de parler de toutes les personnes ayant contribué au développement des nombreux studios au Québec et au Canada. En nous penchant sur des représentants de chacun des cas de figure, nous voulions néanmoins souligner un passionnant héritage en devenir, encore trop méconnu.

Bonne lecture!

Montréal, juillet 2009 